\title{
Tratamentos Figurais e Mobilizações de Registros para a Resolução de Problemas de Geometria
}

\author{
Figural Treatments and Mobilization of Records for the \\ Solving of Geometry Problems
}

\author{
Mariana Moran \\ marianamoranbar@gmail.com \\ Valdeni Soliani Franco \\ vsfranco@uem.br
}

\begin{abstract}
Resumo
Este texto apresenta parte dos resultados de uma pesquisa de doutorado sobre tratamentos figurais e mobilizações de registros que foram realizados durante a resolução de um problema de Geometria por professores de Matemática. Refere-se à investigação da influência dos registros figurais, Material Manipulável (MM), Software GeoGebra (SG) e Expressão Gráfica (EG), na construção e organização do raciocínio dedutivo com apoio visual figural. A teoria utilizada para tal investigação se baseia nos Registros de Representação Semiótica do filósofo e psicólogo francês Raymond Duval. A influência dos tipos de registro figural foi investigada com a participação de 15 (quinze) professores de Matemática da Educação Básica de uma cidade ao norte do estado do Paraná durante a resolução de uma situação problema de Geometria que foi proposta. Com base nos dados obtidos, foi possível concluir que os tratamentos figurais a serem realizados são diferentes, dependendo do tipo de registro que se utiliza, e, da mesma forma, as mobilizações de outros registros também variam conforme o registro figural de partida utilizado.
\end{abstract}

Palavras-chave: Registros Figurais. Tratamentos. Mobilização de Registros. Geometria.

\begin{abstract}
This paper presents some results from a $\mathrm{PhD}$ research on figural treatments and mobilizations of records that were made during the resolution of a Geometry problem by Mathematics teachers. It refers to the investigation of the influence of figural records, Manipulatives (MM), GeoGebra Software (SG) and Graphic Expression (EG), in the construction and organization of deductive reasoning which involved figural visual support. The theory used for this research is based on the Records of Semiotic Representation by the French philosopher and psychologist Raymond Duval. The influence of the types of figural record was investigated with the participation of fifteen (15) Mathematics teachers from Basic Education in a town north of Paraná during the resolution of a Geometry problem-situation. Based on the data obtained, we concluded that the figural treatments to be performed are different depending on the type of record that is used, and likewise the mobilization of other records also vary according to the starting figural record used.
\end{abstract}

Keywords: Figural Records. Treatments. Mobilization of Records. Geometry.

\section{Introdução}

Neste texto, serão apresentados aspectos referentes à influência do tipo de registro figural no tratamento e na mobilização de outros registros durante a resolução de um problema de Geometria elaborado por Duval (1999), e que foi implementado em uma pesquisa de 
doutorado. A figura que compõe o problema a ser resolvido foi representada por meio dos seguintes registros figurais utilizados para a investigação: Material Manipulável (MM), o Software GeoGebra (SG) e a Expressão Gráfica (EG). O problema abordado durante a investigação contempla conteúdos de geometria plana, tais como: segmentos de reta, polígonos e suas áreas, congruência de triângulos, entre outros.

A figura representada por meio do Material Manipulável que foi previamente selecionado, por meio da construção figural no Software GeoGebra e da Expressão Gráfica, foi considerada como tendo representações diferentes - uma em cada um desses registros. Duval (2011, p. 72) explica que "Os registros são sistemas cognitivamente produtores, ou mesmo "criadores", de representações sempre novas".

Este estudo se justifica, sobretudo, por oferecer uma investigação sobre as influências dos tipos de registros figurais durante a exploração de conceitos de Geometria em um contexto de resolução de problema, no que diz respeito às transformações - operações e mobilizações de registros - que podem e devem ser realizadas para que o sujeito obtenha sucesso na aprendizagem e, consequentemente, na conclusão do problema. Além disso, apresenta as possibilidades de representações figurais que podem ser utilizadas no trabalho com conteúdos de Geometria.

Essa pesquisa contou com a participação de 15 (quinze) professores de Matemática da rede pública de ensino de uma cidade ao norte do estado do Paraná. A pesquisa foi submetida e aprovada pelo Conselho de Ética local. A decisão de trabalhar com professores parte do princípio de que esses sujeitos compreendem conceitos básicos de Geometria. Desse modo, é possível fazer a análise das influências dos registros figurais por intermédio desses professores, de tal modo que o objeto de estudo seja os registros, e não o conhecimento dos professores participantes.

Nesse contexto, a Teoria dos Registros de Representação Semiótica ofereceu fundamentações para as investigações desta pesquisa, principalmente porque ela abrange, no conhecimento matemático, aspectos de "referência a um objeto" e de "transformação em outras representações" (DUVAL, 2011). E, além desses aspectos cognitivos, proporciona diversas opções metodológicas para o trabalho com a matemática.

Para a análise dos dados, buscou-se identificar nas falas e nos registros discursivos - língua natural e formal -, os raciocínios mobilizados pelos professores em cada tipo de registro figural, com atenção especial aos aspectos referentes ao tratamento e às conversões. $\mathrm{O}$ problema proposto corresponde a conteúdos de Geometria do Ensino Fundamental e Médio. 


\section{Os Registros de Representação Semiótica em Geometria}

As representações semióticas auxiliam nos sistemas de representação que possuem dificuldades próprias de significado e funcionamento (DUVAL, 2012b). Tratando-se da Geometria, "os objetos que aparecem podem, deste modo, ser diferentes dos tipos de objetos que a situação exige ver" (DUVAL, 2012a). Ou seja, ao visualizarmos somente um desenho (expressão gráfica) de um objeto matemático, por exemplo, um poliedro, não é simples perceber que este objeto tem particulares características como paralelismos, ortogonalidades, entre outros.

Nesse momento, surge a necessidade de conceituar, de acordo com Duval (2011), um registro de representação semiótica. Para o pesquisador, os registros são sistemas semióticos criadores de novos conhecimentos que satisfazem, basicamente, duas condições:

- Produzem representações que permitem acesso e exploração a objetos inacessíveis perceptivelmente ou instrumentalmente.

- Permitem transformações em novas representações.

Duval (2011, p. 98) exemplifica: "Como exemplo de registros, consideramos a língua e as figuras geométricas euclidianas cujas formas podem ser reconhecidas ou construídas materialmente em $3 \mathrm{D} / 3 \mathrm{D}^{1}$,

para considerar um sistema semiótico como um registro, é preciso identificar as
operações de produção de representações que ele permite executar de maneira
original e específica. São essas operações criativas que caracterizam um registro, e
não as regras de combinações válidas, para um sistema formal, ou de signos
utilizáveis, para um código. Assim, cada frase produzida é irredutível às palavras
que ela combina. Duas frases podem empregar exatamente as mesmas palavras e não
ter o mesmo sentido. Por exemplo: "O gato come o rato" e "O rato come o gato"
(DUVAL, 2011, p. 83).

Duval (2012b) explica que, para que um sistema semiótico seja um registro de representação, este deve permitir três atividades cognitivas fundamentais ligadas à semiose ${ }^{2}$ :

- A formação de uma representação identificável como uma representação de um registro dado: deve respeitar regras de utilização, de identificação, de reconhecimento da representação e a possibilidade de sua utilização para tratamentos.

- O tratamento: o tratamento é uma transformação que ocorre internamente ao registro, ou seja, realizam-se operações necessárias para uma questão ou um problema sem sair do

\footnotetext{
1،Toda passagem de uma dimensão a outra representa para o numerador da "fração", $(\mathrm{mD} / \mathrm{nD})$ um salto cognitivo considerável e, analogamente a passagem de uma representação física para uma representação numérica (mudança do denominador)" (DUVAL, 2011, p. 87).

2 "Se é chamada "semiose" a apreensão ou a produção de uma representação semiótica, e "noesis" a apreensão conceitual de um objeto, é preciso afirmar que a noesis é inseparável da semiose” (DUVAL, 2012b, p. 270).
} 
registro inicial. Um exemplo de tratamento bem simples, no registro figural, é a transformação de um triângulo em dois triângulos com a mesma área, conforme a Figura 1:

Figura 1: Transformação de um triângulo em dois triângulos congruentes entre si

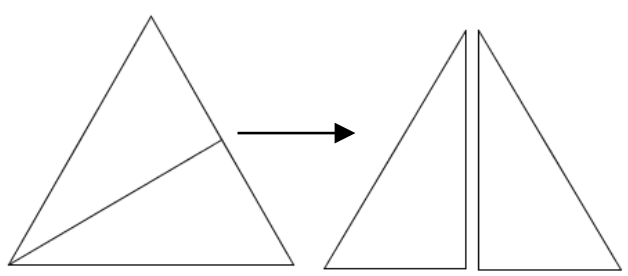

Fonte: Autores

- A conversão: é a transformação de uma representação em outro registro conservando a totalidade ou uma parte somente do conteúdo da representação inicial: "Converter é transformar a representação de um objeto, de uma situação ou de uma informação dada num registro em uma representação desse mesmo objeto, dessa mesma situação ou da mesma informação num outro registro" (DUVAL, 2009, p. 58); “A conversão é uma atividade cognitiva diferente e independente do tratamento" (DUVAL, 2012b, p. 272). Não existe e não podem existir regras de conversão do mesmo modo que existem as regras de tratamento. Como exemplo de conversão, tem-se da língua natural (I) para a expressão algébrica (II) e para a representação gráfica cartesiana (III), representada no Quadro 1:

Quadro 1: Conversão

\begin{tabular}{|c|c|c|}
\hline I & II & III \\
\hline $\begin{array}{l}\text { 1......o conjunto de pontos que tem uma } \\
\text { abscissa positiva }\end{array}$ & $x>0$ & \\
\hline 2......que tem uma ordenada negativa & $\mathrm{y}<0$ & \\
\hline $\begin{array}{l}\text { 3......cuja abscissa e ordenada tem o } \\
\text { mesmo sinal }\end{array}$ & $x y>0$ & \\
\hline 4 & $x y \leq 0$ & \\
\hline $\begin{array}{l}\text { 5......cuja ordenada é superior a abscissa } \\
\text { (a reta y = x sendo já traçada no gráfico) }\end{array}$ & $y>x$ & \\
\hline $\begin{array}{l}\text { 6......cuja ordenada é superior a abscissa } \\
\text { (a reta } y=x \text { não sendo traçada no gráfico) }\end{array}$ & $y>x$ & \\
\hline 7......cuja ordenada é igual a abscissa & $y=x$ & \\
\hline 8......cuja ordenada é oposta a abscissa & $y=-x$ & \\
\hline
\end{tabular}

Fonte: Duval, 2012b, p. 274 
Desse modo, quando o aluno é capaz de coordenar espontaneamente os vários registros de representação de um mesmo objeto, significa que ocorreu de fato uma aprendizagem de determinado conceito ou conteúdo (DUVAL, 2012b).

\title{
O uso dos Materiais Manipuláveis, do Software GeoGebra e das Expressões Gráficas como Registros Figurais
}

Neste trabalho, utiliza-se o Material Manipulável, o Software GeoGebra e a Expressão Gráfica para representar figuras geometricamente. Considera-se essas três formas de "ver" as figuras como registros figurais, ou seja, como sistemas semióticos que permitem abstrações cognitivas utilizáveis em resolução de problemas ou em reconhecimentos de propriedades geométricas.

Entende-se por Material Manipulável tudo que pode ser manipulado pelo sujeito:

\begin{abstract}
Alguns não possibilitam modificações em suas formas: é o caso dos sólidos geométricos construídos em madeira ou cartolina, por exemplo, que por serem estáticos permitem só a observação. Outros já permitem uma maior participação do aluno: é o caso do ábaco, do material montessoriano (cuisenaire ou dourado), dos jogos de tabuleiro (LORENZATO, 2006, p. 18-19).
\end{abstract}

Desse modo, será trabalhado com uma atividade proposta para utilização desse material, que permitirá ao sujeito modificações e operações visuais que possibilitem o tratamento figural e a mobilização de outros registros. Será utilizado também o Software GeoGebra para representar figuras geométricas.

O uso de softwares permite uma mobilidade, pelo sujeito, de operações com as figuras, por exemplo, o arrastar de um vértice, a aproximação de um polígono inscrito a uma circunferência por meio do comando controle deslizante em função do número de lados do polígono, entre outras interações.

É primordial destacar também que, com base na leitura de registro figural de Raymond Duval, admitem-se, nesta pesquisa, as figuras realizadas por meio da Expressão Gráfica como um registro figural. Entende-se por Expressões Gráficas as figuras construídas com o uso de materiais que auxiliem a construção de desenhos e principalmente figuras em geral, como, por exemplo, régua, transferidor, esquadro, compasso, lápis etc. As figuras assim construídas formam imagens passíveis de comunicar uma ideia, um conceito ou um pensamento.

De acordo com Duval (2011), as figuras na geometria apresentam três características que lhes conferem um poder cognitivo específico: têm um valor intuitivo, permitem um 
reconhecimento praticamente imediato dos objetos e podem ser construídas com régua, com compasso ou com um software.

\section{Metodologia}

A metodologia da pesquisa segue uma abordagem qualitativa, de cunho interpretativo. A pesquisa foi realizada com 15 (quinze) professores de Matemática da Educação Básica da Rede Estadual de Ensino de uma região ao norte do estado do Paraná, Brasil.

A atividade foi aplicada individualmente com cada professor participante. Os pesquisadores tentaram, assim como sugere Alves-Mazzotti e Gewandsznajder (2004), ser capazes de estabelecer uma relação de confiança com os sujeitos, ser bons ouvintes, formular perguntas, ter familiaridade com as questões investigadas e ter flexibilidade para se adaptar a situações inesperadas.

Para as respostas, foram solicitadas justificativas oralmente e por extenso, na forma de língua natural e formal ${ }^{3}$, pois Duval (2011, p. 99) afirma que "Pensar em matemática mobiliza sempre pelo menos dois registros", e, "em geometria, mobilizamos a linguagem e a visualização".

No decorrer das análises, fragmentos das entrevistas e das respostas escritas dos professores são destacados para justificar as conclusões realizadas. As informações analisadas estão subdivididas em unidades de análise com a seguinte prescrição: A1 e A2. E os professores colaboradores, indicados por P1, P2, P3, e assim por diante, até P15. Usa-se fonte em itálico sem aspas para as falas e fonte comum com aspas para a escrita dos professores entrevistados.

O objetivo da atividade aplicada foi explorar propriedades por meio dos registros figurais numa progressão de manipulações práticas ou concretas até seus registros discursivos. Duval (2011, p. 140) afirma que "a questão chave da interpretação é então aquela das transferências internas de um modo a outro e de um registro a outro".

O problema envolve conceitos de geometria e é possível de ser resolvido utilizando tanto o Material Manipulável quanto o Software GeoGebra e a Expressão Gráfica. Estes foram previamente selecionados e disponibilizados, para o momento da aplicação, pelos pesquisadores aos professores colaboradores para resolução.

\footnotetext{
${ }^{3}$ A língua natural se refere à língua materna do Brasil (o português), enquanto a língua formal faz referência à simbologia utilizada nas deduções matemáticas.
} 
Sendo assim, os professores participantes foram divididos em 3 (três) grupos de 5 (cinco) pessoas cada, onde as ordens de apresentação do problema nos diferentes registros foram variadas, a saber:

$$
\begin{aligned}
& \text { Grupo 1: } \mathrm{SG} \rightarrow \mathrm{EG} \rightarrow \mathrm{MM} \\
& \text { Grupo 2: } \mathrm{MM} \rightarrow \mathrm{SG} \rightarrow \mathrm{EG} \\
& \text { Grupo 3: } \mathrm{EG} \rightarrow \mathrm{MM} \rightarrow \mathrm{SG}
\end{aligned}
$$

Mesmo quando o professor participante resolvia, corretamente ou não, o problema com o primeiro registro, foram apresentados a ele também o segundo e o terceiro, nessa ordem, de modo a tornar possível investigar se estes registros influenciariam na resposta dada anteriormente, fazendo-o, talvez, repensar a sua solução e também verificar se surgiriam novas ideias para a resolução do problema.

A aplicação da atividade contou com gravação de áudio e registro da resolução de cada problema em uma folha entregue pelos pesquisadores. Até mesmo para os professores que não chegaram à solução, houve o pedido de registro discursivo sobre o máximo de conclusões retiradas a respeito do problema com base nos tipos de registros figurais oferecidos para a resolução. Além de permitir analisar os registros mobilizados pelos professores para a resolução dos problemas, esta estruturação dos dados permitiu perceber os tratamentos efetivados e a interpretação figural realizada pelo professor com base em cada tipo de registro oferecido (MM, SG, EG).

As resoluções individuais dos professores participantes foram analisadas com base em seus raciocínios expostos na forma de registro discursivo (língua natural e formal) levando em consideração os sucessos, hesitações e fracassos, com atenção especial aos tratamentos e mobilizações de outros registros. Usou-se a produção oral e escrita para a análise, porque Duval (2011, p. 105) afirma que "a produção oral e escrita não têm os mesmos papéis na tomada de consciência [...] das unidades de sentido matematicamente pertinentes em uma representação". Ou seja, pode parecer simples falar sobre determinado assunto oralmente, mas, diante da necessidade de escrever tal raciocínio, surgem questões manifestadas pela tomada de consciência e organização da escrita.

\section{Atividade e Resultados}

A atividade a seguir foi implementada com professores de Matemática da Educação Básica. Sendo assim, neste texto, será apresentada essa atividade e suas soluções para fazer uma análise a respeito de alguns aspectos relacionados aos tratamentos e mobilizações figurais. 
Atividade $^{4}$ : Mostrar a igualdade das áreas 1 e 2, qualquer que seja a posição do segmento $\overline{\mathrm{AB}}$ (DUVAL, 1999, p. 157).

Figura 2: Registros figurais: Atividade 1

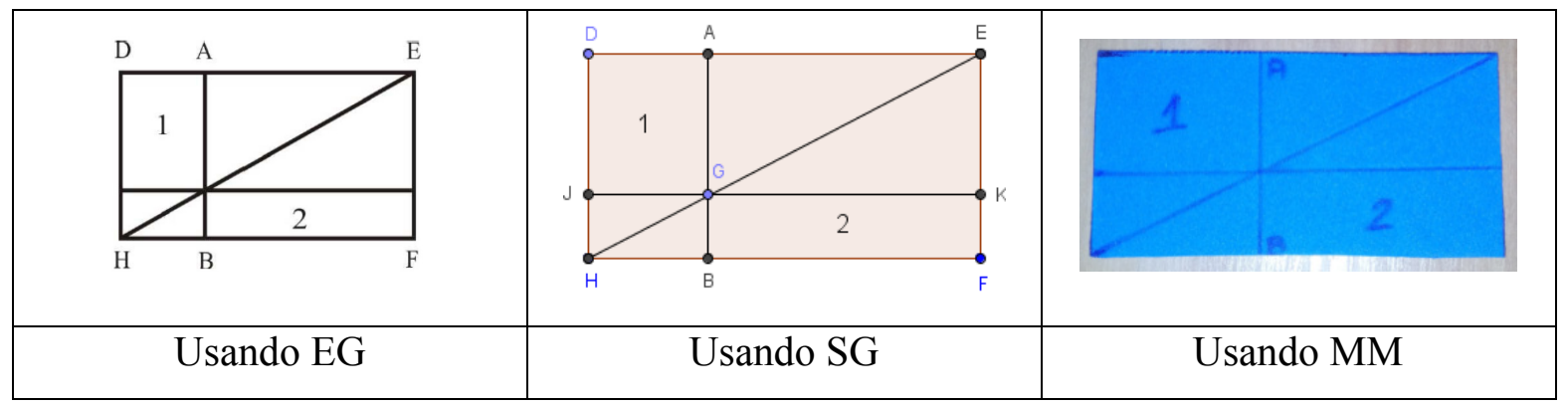

Fonte: (DUVAL, 2012a, p. 129) e Autores

Este problema pode ser resolvido por uma modificação figural do tipo mereológica, fazendo uma operação de reconfiguração que consiste no fracionamento da figura inicial em subfiguras. Neste caso, por congruência entre os triângulos GÂE $\equiv \mathrm{E} \widehat{K} G$ e entre $H \hat{J} G \equiv \mathrm{G} \hat{B} \mathrm{H}$, conclui-se, a igualdade entre as áreas dos quadriláteros 1 e 2, conforme a Figura 3.

Figura 3: Solução da Atividade 1

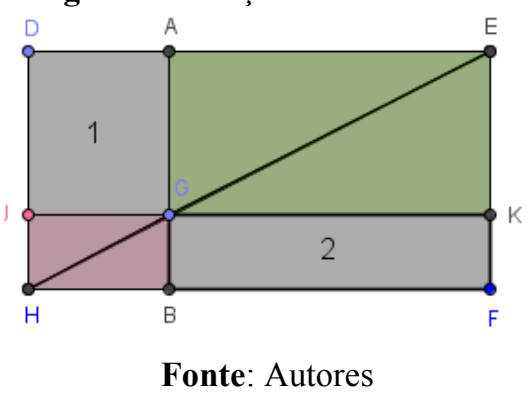

O objetivo principal desta atividade foi, por meio da variação do tipo de registro figural, analisar a compreensão, ou seja, o alcance em termos de autonomia e progressão do sujeito durante a resolução do problema. Para isso, esta análise foi dividida em duas unidades:

A1: Se houve tratamento figural para resolver o problema: caso tenha havido, verificar se ocorreram variações cognitivas (interação de tratamento figural com tratamento discursivo).

É importante analisar se, durante o contato com os diferentes tipos de registros figurais, ocorre a possibilidade de tratamentos figurais e também a simultaneidade com o tratamento discursivo. Duval (1999) explica que os tratamentos parecem vir de leis de organização

\footnotetext{
${ }^{4}$ No início da aplicação da atividade, foi disponibilizado aos professores colaboradores lápis, borracha, caneta, régua, tesoura, cola e folha de resposta. Os rascunhos foram realizados em uma folha de resposta que foi entregue.
} 
figural de percepção visual e, como consequência do entendimento, ocorre um discurso teórico usado para se comunicar, no caso, as variações cognitivas.

Para resolução desta atividade, era extremamente necessário que se efetuasse tratamentos figurais em busca de demonstrar dedutivamente a igualdade das áreas 1 e 2. Dessa forma, como justificativa para a solução, o tratamento discursivo seria consequência do tratamento figural realizado.

A seguir, a análise realizada em cada grupo:

Grupo 1: Neste grupo, iniciou-se com o SG e todos os professores efetuaram tratamento figural no momento em que transladavam o segmento $\overline{A B}$, por meio do ponto $\mathrm{G}$ de intersecção deste com a diagonal do retângulo, conforme a Figura 4, de modo a observar (visualmente) que as áreas 1 e 2 continuavam as mesmas.

Figura 4: Modificações posicionais no retângulo

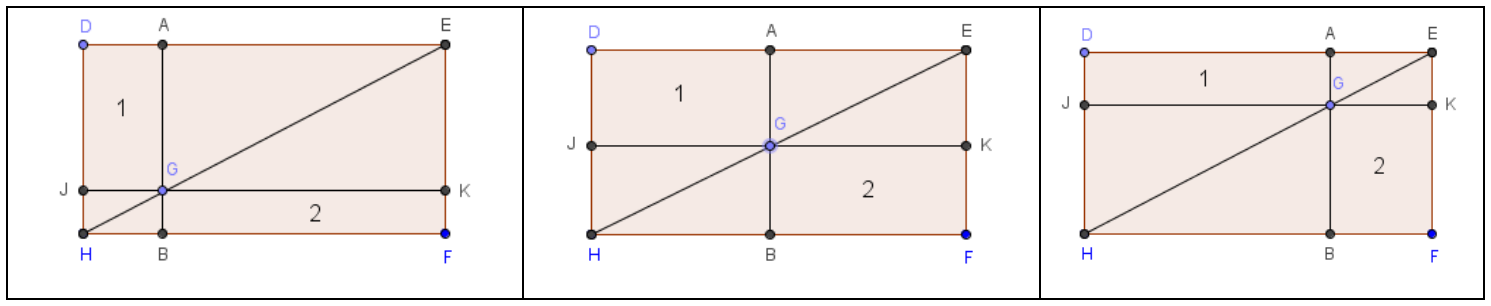

Fonte: Autores

É interessante notar que a passagem deste ponto (ponto $G$ ) pelo ponto médio da diagonal do retângulo possibilitava aos professores intuir que as medidas das áreas 1 e 2 realmente eram iguais.

Ainda neste grupo de professores, foi constatado que nenhum professor efetuou tratamento figural com o registro da EG, e somente 1 (um) professor realizou tratamento figural com o MM, fazendo o recorte da área 1, de modo que suas partes pudessem ser sobrepostas à área 2, verificando, assim, a igualdade das áreas, conforme a Figura 5.

Figura 5: Recorte da parte 1 para sobrepor na parte 2

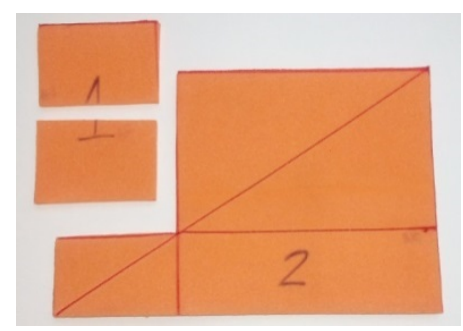

Fonte: Autores 
Grupo 2: Neste grupo, o registro inicial dado foi o Material Manipulável. Com este registro, 3 (três) professores realizaram um tratamento figural, e todos da mesma forma. Houve variação cognitiva no sentido de que estes professores efetuaram um tratamento discursivo para explicar o tratamento figural que haviam feito, como relata $\mathrm{P} 8$ :

P8: "Usando o material manipulável foi possível mostrar a equivalência das partes recortando 1 e sobrepondo em 2".

No SG, 3 (três) professores transladaram o segmento $\overline{A B}$ em busca de uma solução para o problema, porém somente 2 (dois) deles relataram o tratamento realizado. Já no uso do registro da EG, nenhum professor efetuou qualquer tipo de tratamento figural para tentar encontrar uma solução para o problema.

Grupo 3: Neste momento da aplicação da sequência, esta atividade iniciou-se com o registro figural na forma de Expressão Gráfica. Com este registro, 1 (um) professor efetuou tratamentos figurais no desenho e descreveu, utilizando raciocínio dedutivo por meio de linguagem natural e formal, os tratamentos efetuados na figura.

Em seguida, ao disponibilizar o MM, todos os professores efetuaram tratamentos figurais no material, porém o professor que já havia resolvido o problema dedutivamente o fez de forma diferente dos demais. Ou seja, 4 (quatro) professores recortaram a área 1 de modo que ela pudesse ser sobreposta à área 2 ou vice-versa, e, dessa forma, concluíram a igualdade das áreas. Já o outro professor recortou todas as subfiguras da figura (retângulos e triângulos) e fez a sobreposição dos triângulos congruentes para concluir que as áreas 1 e 2 são iguais, conforme a Figura 6:

Figura 6: Tratamento figural no MM

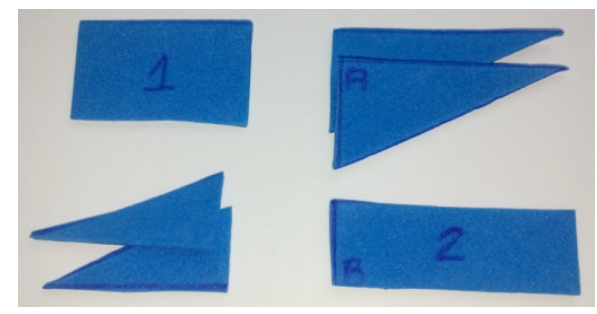

Fonte: Autores

Então, com o SG, houve a interação de 3 (três) dos 5 (cinco) professores efetuando a translação do segmento $\overline{A B}$ e descrevendo discursivamente o que foi realizado, também atentos ao ponto médio da diagonal do retângulo, conforme abaixo: 
P12: "Ao observar a figura e mover o ponto de intersecção, consegui visualizar de imediato o movimento e a semelhança das áreas 1 e 2 , com base no ponto médio".

Considerações: É interessante observar que os tratamentos a serem realizados em cada registro foram diferentes. Destacando:

- MM: manuseio, recorte e sobreposição de partes.

- SG: translação do segmento $\overline{A B}$ até o ponto médio da diagonal.

- EG: separação das subfiguras da figura.

Sendo esta última possível de ser realizada também nos outros dois registros.

Por fim, com base nas observações e dados dos grupos 1, 2 e 3, foi possível notar que, no que se refere a tratamentos que podem ser efetuados nas figuras, o Software de Geometria e o Material Manipulável possibilitaram mais transformações desse tipo, suscitando a criatividade e, consequentemente, o uso do raciocínio lógico em busca da solução para o problema. Esses registros também encaminharam os sujeitos para que estes pudessem discursar a respeito de suas ideias, proporcionando uma organização estruturada do pensamento, conforme constatado nos registros discursivos desses professores colaboradores.

A2: Se houve mobilização de um segundo registro para resolver o problema: caso tenha havido, verificar se ocorreu discriminação das unidades significantes dos registros de partida no registro de chegada.

Com relação à mobilização de um segundo registro na tentativa de solucionar o problema proposto, Duval (2011) explica que, durante uma compreensão matemática, o sujeito mobiliza sempre, pelo menos, dois registros de representação semiótica. Buscou-se, ao analisar neste trabalho a mobilização de outros registros, identificar a possível compreensão do problema, bem como de sua solução.

Para resolução desta atividade, era necessário que se mobilizasse o registro da língua formal com o intuito de demonstrar a congruência entre os triângulos GÂE e E $\widehat{K} G, H \hat{J} G$ e $G \hat{B} H$. Tal mobilização deve ser feita por meio da identificação das unidades significantes do registro de partida (figural), bem como os lados e os ângulos congruentes, representados também no registro de chegada (língua formal ou simbólica).

A seguir, a análise de cada grupo:

Grupo 1: Do total de 5 (cinco) professores deste grupo, 4 (quatro) mobilizaram outro tipo de registro durante o uso do SG. Seguem: 
- P1 fez uma passagem do registro figural para o registro numérico no momento em que estabeleceu uma razão entre os lados das áreas 1 e 2.

- P2 fez uma passagem do registro figural para o registro da língua formal discriminando as unidades significantes de cada registro e utilizando um raciocínio dedutivo que solucionou o problema.

P2: "O segmento $\overline{\mathrm{EH}}$ é a diagonal da figura $\mathrm{DEFH}$, então o $\triangle \mathrm{DEH} \approx \triangle \mathrm{EFH}$. $\mathrm{A}$ diagonal $\overline{E G}$ do retângulo AEIG o divide em $2 \Delta$ s equivalentes. O mesmo ocorre no retângulo BGJA. Logo, temos que as partes 1 e 2 são equivalentes".

- P3 fez uma passagem do registro figural para o registro numérico quando utilizou a ferramenta "polígono", selecionou os dois retângulos e, com a ferramenta "área", calculou a área de cada um deles e comprovou que eram iguais.

- P4 fez uma passagem do registro figural para o registro da língua natural descrevendo o raciocínio que o fez concluir o problema, por extenso e em linguagem natural.

No uso da EG, obteve-se 2 (duas) mobilizações de outros registros. Seguem:

- P1, que permaneceu na passagem feita anteriormente no SG, do registro figural para o registro numérico, estabelecendo razões e proporções entre as áreas.

- P3, que permaneceu na passagem do registro figural para o registro numérico medindo os lados dos polígonos 1 e 2 e calculando suas áreas.

No último registro entregue, o MM, obteve-se, também, 2 (duas) mobilizações, ambas do registro figural para o registro numérico feitas por $\mathrm{P} 1$ e $\mathrm{P} 3$. Vale enfatizar que $\mathrm{P} 1$ mudou do raciocínio de razão e proporção para o cálculo da área de cada polígono de modo a verificar se eram iguais, enquanto P3 permaneceu no cálculo das áreas conforme os registros anteriores.

Grupo 2: No grupo 2, a atividade se iniciou com o registro figural na forma de Material Manipulável. Para resolver esta atividade com este recurso, somente 1 (um) professor efetuou uma mobilização de registro, sendo esta do registro figural para o registro numérico, medindo os lados dos polígonos 1 e 2 de modo a calcular suas áreas e verificar a igualdade.

O próximo registro disponibilizado foi o SG. Neste registro, outros 2 (dois) professores efetuaram uma mobilização do registro figural para o registro da língua formal. Estes discriminaram as unidades significantes da figura (vértices, diagonal, segmentos de reta, retângulos), em seguida utilizaram a linguagem simbólica para representar cada uma dessas 
unidades significantes (notação para vértices, segmentos, congruência, equivalência, igualdade), e um deles concluiu formalmente a demonstração da igualdade das áreas 1 e 2 de maneira correta.

Ao disponibilizar o registro na forma de EG, 3 (três) professores, inclusive o professor que já havia resolvido o problema dedutivamente, sugeriram o uso de régua para medir os lados dos polígonos 1 e 2 e verificar a igualdade de suas áreas, ou seja, mobilizaram o registro figural para o numérico.

Grupo 3: Neste grupo, foi apresentado, primeiramente, o registro figural na forma de Expressão Gráfica. Neste registro, 4 (quatro) professores mobilizaram outros registros de representação, sendo 3 (três) do registro figural para o numérico e 1 (um) do registro figural para o registro da língua formal. A mudança do figural para o numérico foi feita por meio de anotações de medidas e cálculo de área (2 (dois) professores) e pelo uso de razão e proporção (1 (um) professor):

P11: "Utilizando a expressão gráfica para a resolução, usei a régua para verificar as dimensões (comprimento $\mathrm{x}$ largura) das partes 1 e 2 e calculei as duas áreas demonstrando a igualdade entre elas".

Após disponibilizar o MM, nenhum professor efetuou qualquer tipo de mobilização do registro figural para outro tipo de registro, ficando restritos ao tratamento neste material. Quando foi apresentada a mesma figura, porém no SG, houve manifestação de 2 (duas) transformações de registros: P11, que havia feito a mudança do figural para o numérico na EG, fez no SG uma mobilização do figural para a língua formal; e P13, que havia sugerido o uso de razão e proporção na EG, fez uso do cálculo das áreas 1 e 2 usando as ferramentas que o software disponibiliza.

Considerações: Por meio destas informações compiladas, é possível perceber que o registro da EG foi o que proporcionou maior intenção de mobilização em relação aos outros registros de representação, porém quase sua totalidade foi do registro figural para o registro numérico. Duval (2011) afirma que, para aprender, os sujeitos devem trabalhar sem recorrer primeiro a aspectos métricos, ou seja, é necessário haver um maior apelo à demonstração, principalmente em atividades como essa.

$\mathrm{Na}$ interação do professor com o SG, foi possível perceber também várias mobilizações para outros registros, sendo exageradamente maior - 8 (oito) para 3 (três) - do que no contato com o MM. O SG proporcionou o maior número de mobilizações do registro figural para o 
registro da língua formal (linguagem simbólica); já o $\mathrm{MM}$ não provocou nenhuma transformação deste tipo, sendo todas do figural para o numérico, conforme descrito e observado anteriormente.

\section{Considerações finais}

O uso da Teoria dos Registros de Representação Semiótica em Geometria foi, sem dúvida, primordial para esta investigação, direcionando o modo como foram elaborados os instrumentos de pesquisa e as conclusões descritas nas análises.

Conforme os raciocínios explicitados pelos professores no decorrer das aplicações do problema que levou à análise dos dados, é possível concluir que:

* quanto ao uso de tratamento figural para resolver o problema, em cada tipo de registro figural - Materiais Manipuláveis, Softwares Geométricos e Expressões Gráficas -, os professores realizavam tratamentos diferentes. Tal fato reforça a ideia de que é importante que o sujeito conheça as várias representações para um mesmo objeto matemático, pois cada uma delas desenvolve aspectos cognitivos e matemáticos particulares. Em conformidade com a Teoria dos Registros de Representação Semiótica, é o conhecimento das várias representações para um mesmo objeto que possibilita a construção do conhecimento. Na atividade realizada, o SG se destacou ao proporcionar maior interação do sujeito na realização de tratamentos figurais. Também houve destaque do MM, que encaminhou os sujeitos para que discorressem melhor sobre suas ideias, direcionando-os para uma organização estruturada de pensamento, conforme observado nos registros discursivos dos professores colaboradores.

quanto à mobilização de um segundo registro para resolver o problema, os registros figurais na forma de $\mathrm{EG}$ se destacaram por induzir os professores colaboradores à transformação do registro figural para o registro numérico. É importante ressaltar que tal problema foi proposto com a intenção de que os sujeitos, ao ter contato com os registros, utilizassem deduções matemáticas, pois o uso de cálculos serve para verificar e não demonstrar resultados. Com relação ao $\mathrm{SG}$, este foi o registro que possibilitou o maior número de conversões do tipo figural para língua formal. Talvez isso se deva à dinâmica que a figura possui quando representada neste tipo de registro figural.

Desse modo, esta pesquisa mostra que, ao utilizar diferentes registros figurais na resolução de problemas de geometria, fatores referentes aos tratamentos e às mobilizações de registros são modificados, gerando consequências diretas na busca da solução do problema. Do ponto de 
vista matemático, Duval (2011) explica que a solução do problema é o que demonstra os diferentes conhecimentos que permitem resolvê-lo. No entanto, do ponto de vista cognitivo, são analisados os processos que permitem reconhecer os conhecimentos matemáticos a serem empregados, "Pois não serve de nada se lhe explicamos a solução, se você não vê como poderia pensar sobre ele" (p. 41).

\section{Referências}

ALVES-MAZZOTTI, Alda J.; GEWANDZNAJDER, Fernando. O Método nas Ciências Naturais e Sociais: Pesquisa Quantitativa e Qualitativa.São Paulo: Pioneira Thomson Learning, 2004.

DUVAL, Raymond. Semiosis y pensamento humano: Registros semióticos y Aprendizajes intelectuales. Tradução: Myriam Vega Restrepo. Cali, Colombia: Universidade del Valle, 1999.

DUVAL, Raymond. Semiósis e Pensamento Humano: Registros semióticos e aprendizagens intelectuais (Fascículo I). Tradução: Lênio Fernandes Levy e Marisa Rosâni Abreu da Silveira. São Paulo: Editora Livraria da Física, 2009.

DUVAL, Raymond. Ver e ensinar a matemática de outra forma: entrar no modo matemático de pensar: os registros de representação semióticas. Org.:Tânia M. M. Campos; tradução: Marlene Alves Dias. 1ed. São Paulo: PROEM, 2011.

DUVAL, Raymond. Abordagem cognitiva de problemas de geometria em termos de congruência.Tradução: Méricles Thadeu Moretti. Revista Eletrônica de Educação Matemática - Revemat, v.07, n.1, p.118-138, 2012a.

DUVAL, Raymond. Registros de representação semiótica e funcionamento cognitivo do pensamento.Tradução: Méricles Thadeu Moretti. Revista Eletrônica de Educação Matemática - Revemat, v.07, n.2, p. 266-297, 2012 b.

LORENZATO, Sergio. Laboratório de ensino de matemática e materiais didáticos manipuláveis. In: (Org.). O Laboratório de Ensino de Matemática na Formação de Professores. Campinas, SP: Autores Associados, 2006. 